\title{
Upper third auricular reconstruction surgery of cauliflower ear following auricular perichondritis: a
}

\section{case report}

\begin{abstract}
Cauliflower ear is the deformity result of necrosis of the cartilage and which is caused by several etiologies. One of the etiology was perichondritis and as the disease progress, abscess will developed and peel the perichondrial layer off the cartilage and resulting the necrosis and deformity of the auricle. This paper will discuss about male patient who developed cauliflower ear after had several episodes of perichondritis. This paper will further discuss about the reconstruction surgery for the deformity.
\end{abstract}

Keywords: Cauliflower ear, reconstruction surgery, perichondritis
Volume 12 Issue 3 - 2020

\section{Reksodiputro MH, Sriyana AS, Reksodiputro LA, Mufida T \\ Department of Otorhinolaryngology Head \& Neck, Indonesia}

Correspondence: Reksodiputro MH, Department of Otorhinolaryngology Head \& Neck, Faculty of Medicine University of Indonesia/Dr. Cipto Mangunkusumo Hospital, Jakarta, Indonesia, Email citamirta@yahoo.com

\section{Introduction}

Cauliflower ear is a condition of acquired deformity of the auricle. The most common cause of cauliflower ear is repeated blunt trauma to the ear or continuous abrasion of the pinna. It is commonly seen among wrestlers $^{1,2}$ It can also be caused by perichondritis, inflammatory condition of the auricular cartilage. Staphylococcus aureus and Pseudomonas aeruginosa are the most common pathogen involved. ${ }^{3}$ In the long term, destruction of auricular cartilage further causes abscess and peeling of perichondrial layer off the cartilage. The perichondrial layer delivers nutrition to the avascular cartilage, therefore in the condition where the perichondrial layer got peeled off the cartilage, the cartilage became necrotic and cauliflower ear deformity occurs. ${ }^{4}$ It is important to manage early features of perichondritis with antipseudomonal agents and early surgical intervention when abscess is formed. Although, with timely management of perichondritis, cauliflower ear may still occurs.

Cauliflower ear causes cosmetic problems including protrusion and distortion of the ear. Pain or discomfort associated to the deformity sometimes occur. The deformity can also spread to the ear canal and conductive hearing loss may happens. ${ }^{2}$ The exact prevalence of cauliflower ear hasn't been described in literature. In general, the prevalence is high in impact sport's athletes such as wrestlers, reaching approximately $40 \%$. The risk is higher in athletes who do not use protective headgear. ${ }^{5}$ It is also more prevalent in male than female. ${ }^{1}$ On the other hand prevalence of cauliflower ear in nonathletes is still unknown, since studies on the topic are very scarce.

Reconstruction of the auricle remains a challenge in plastic surgery because of the complex anatomy of the cartilage and overlying skin. In general, the outcome of auricular reconstructive surgery is to remain aesthetic subunit of the auricle. Optimal auricular reconstruction is selected based on the type and location of the deformity. Cauliflower ear is characterized by thickening and irregular projection of the auricle, therefore the goal of cauliflower ear reconstructive surgery is to restore normal ear contours. To achieve this goal, various surgical correction technique have been proposed. ${ }^{5}$ This paper will further discuss the reconstruction surgery of cauliflower ear, specifically on upper third auricular defect. ${ }^{6}$

\section{Case report}

Reported one case of 21 years old male with chief complaint deformity of his right auricle since 6 years ago started with existence of furuncle on his right auricle. The furuncle burst and the infection spread. Patient went to general practitioner and had a medication until the furuncle resolved. There were intermittent pain, edema, and hyperemic in the right auricle. The edema improved but the auricle became wrinkled and shrank. There is no hearing disturbance nor ear discharge. There was no history of congenital auricular deformities, trauma, piercing, recurrent pressure with helmet, diabetes mellitus, nor other systemic disease. Patient felt unconfident with his right auricle. Physical examination showed there was deformity resembling cauliflower in the right auricle. There was shortening of the helix and antihelix cartilage, also the triangular fossa was wrinkled. In middle ear examination, his right auricle had intact tympanic membrane with no discharge and deformities.

Auriculoplasty was performed to the patient. Incision was done at the helix, and then undermined to superior and posteriorly and remains $1,5 \mathrm{~cm}$ from the retroauricle sulcus. The necrotic cartilage was removed and soft tissue debridement of the surrounding area was performed. Costal cartilage harvested from the seventh rib about $5 \mathrm{~cm}$ length. The cartilage was sculpted to form upper third auricle which was detailed to form helix and triangular fossa. The frame was inserted and sutured to the remain ear cartilage. Drain was inserted and the wound was continuously sutured with prolene 4.0.

The patient was hospitalized and the vacuum drain remains until 5 days with intermittent suctioning to maintain negative pressure. The medical management was ceftriaxone $1 \mathrm{x} 2 \mathrm{~g}$ IV for prophylactic antibiotic and gauze changing everyday. One week after the operation the wound was still in good healing process and the suture remains. There are no hyperemic nor signs of infection. The sulcus formation not yet visualized. Two weeks after the surgery, the suture was removed and there are no signs of necrosis nor exposed cartilage. There are no signs of inflamation nor infection. The sulcus between heliks and triagular fossa starts to formed. One month after surgery the wound was healed and had a good contour helix and triangular fossa. The patient was electively planned to do the second stage surgery for elevating the auricle after the first stage operation (Figures 1-3). 


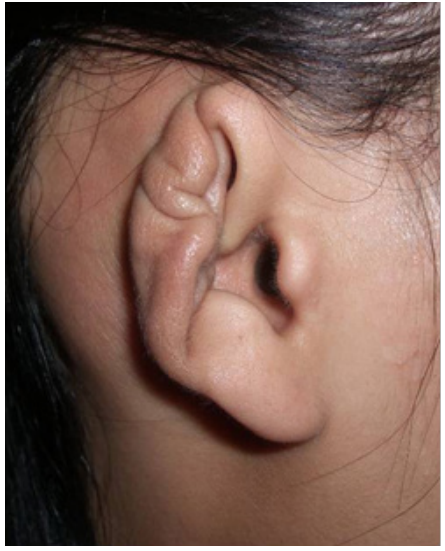

Figure I Pre operation photo.

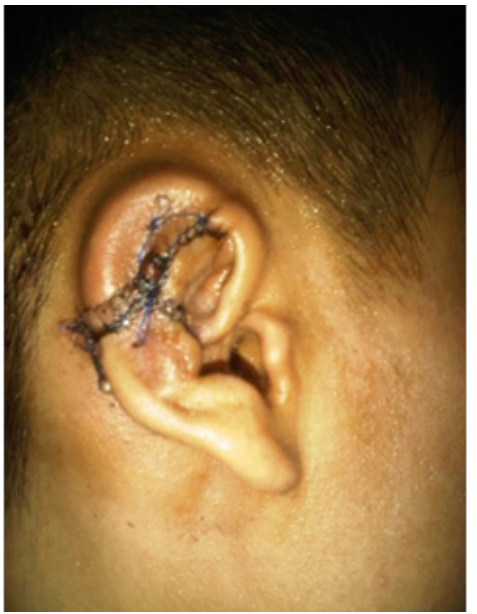

Figure 2 Post operation day 8.

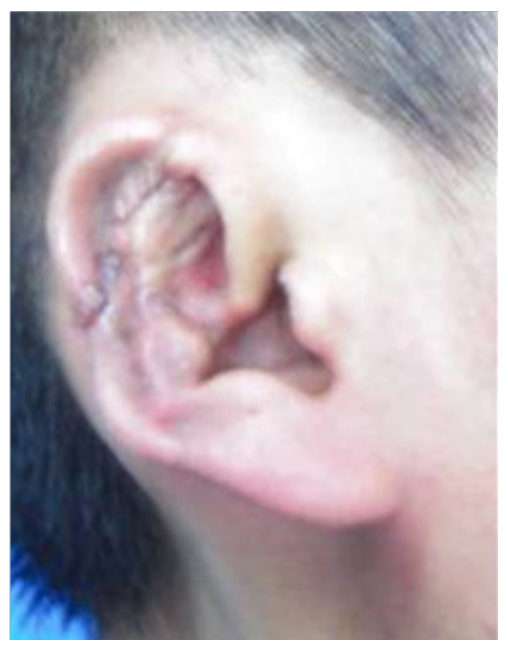

Figure 3 One month post operation.

\section{Discussion and review of literature}

Patient, 21 years old male, came with auricular deformity complaint. He had several episodes of perichondritis for the last six years and did not seek for medical attention. The auricle became smaller, wrinkled and deformed resembling cauliflower. In the literature cauliflower ear is the result of necrosis of the cartilage, soft tissue contraction which caused deformity of the auricle aesthetic subunit (Figure 4). One of the etiology was perichondritis and as the disease progress, abscess will developed and peel the perichondrial layer off the cartilage resulting necrosis and deformity of the auricle. Patients with cauliflower ear deformity have two concerns: the appearance and pain or discomfort associated with the deformity. The ultimate goal in surgical treatment is to restore the contours of the normal auricle. Achievement of this goal represents a challenging problem by all means. To date, very few cases of surgical correction have been described and range from shaving, partial resection and resection with graft reconstruction. The pathophysiology of the deformity starting by a subperichondrial hematoma and ending up in a deformed cauliflower-like appearance is of paramount importance to plan surgical treatment. ${ }^{12,13}$

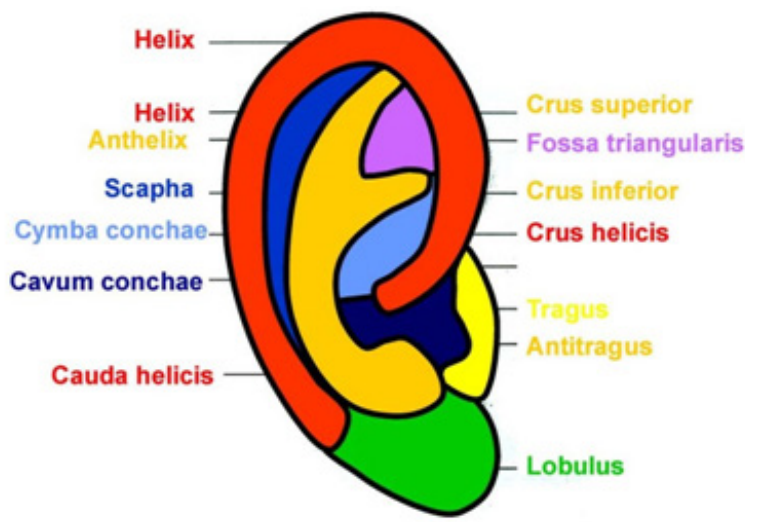

Figure 4 Aesthetic Subunit of The Auricle. ${ }^{6}$

Auricular reconstruction surgery was performed to reconstruct his right auricle. He had the first stage reconstruction of the upper third auricular surgery. Costal cartilage harvested from the seventh rib about $5 \mathrm{~cm}$ and sculpted to form helix and triangular fossa. The frame was inserted and sutured to the remain auricular cartilage. There are little amount of literature that describe the auricular reconstruction surgery for the cauliflower. In this case the cauliflower made up the upper third auricular defect. The reconstruction surgery could be based as the classification of auricular defect made by Weerda, 1980. The upper third auricular reconstruction surgery consist of several techniques depends on the defect size. Another methods like helical chondrocutaneous advancement flap and bipedicled tubed flap are used for smaller defect and it will reduce the auricle size. Medium-sized defects can also be closed with composite grafts from the contralateral ear. The graft should be half the defect size to allow for symmetry between the two ears. The large defect as in this case, requires the use of cartilage, temporoparietal flap, and a skin graft for reconstruct the upper third. We used costal cartilage graft for fabricating the framework and inserted in the subcutaneous pocket that made at the temporal region (Figures $5 \& 6$ ). ${ }^{7-16}$

The first stage operation was implantation of the cartilage framework. The skin incised about 3-4 mm lower than the marked height of the stump. ${ }^{7,8}$ Subcutaneous pocket is developed, about 1 $\mathrm{cm}$ larger than the height of the superior helical margin and the post auricular stump skin is sutured to the lower margin of the incision. The framework is attached using 5.0 braided suture and inserted to the pocket. Closure of the skin wound, vacuum drainage, and mattress sutures (Figures 7-10). ${ }^{7}$

The patient was planned for second stage surgery. There are few technique for elevating the auricle. In one literature stated that the Nagata method is better for the second stage surgery other than 
Ombredanne's and Crikelair's, but there are no evidence based that support this statement. Nagata technique begin with Incision of full thickness skin flap pedicled on the helix about $8-10 \mathrm{~mm}$ in width is elevated above the helix and above the hair follicles in the direction of helix, leaving some fibrous tissue on the helical cartilage. Next the entire post auricular surface and the mastoid surface covered with thick split- or full thickness skin graft taken from thorax donor site, the groin area or buttocks. The skin graft is secured with sutures and fibrin glue (Figure 11).

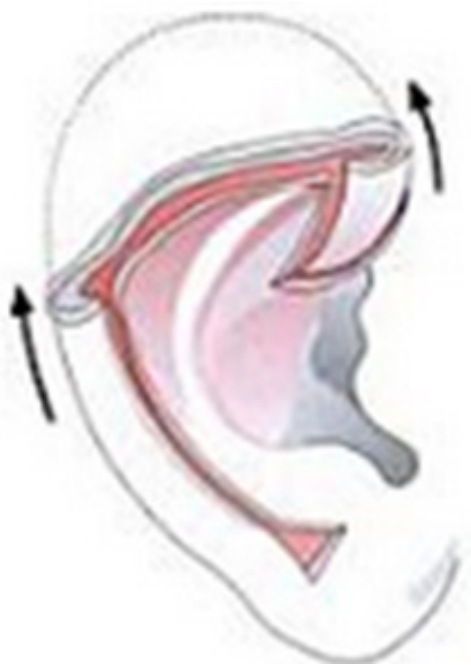

Figure 5 Coverage of a superior defect with posterior and anterior helical sliding flap. ${ }^{?}$

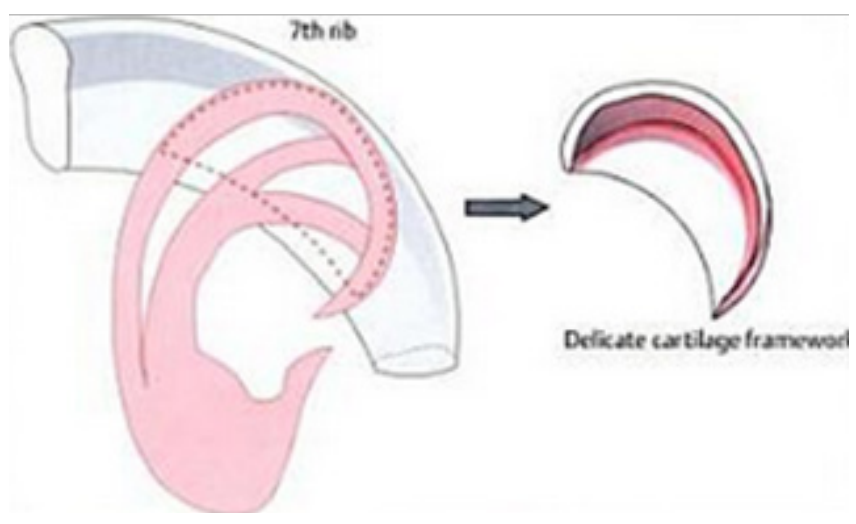

Figure 6 Carving the framework. ${ }^{7}$
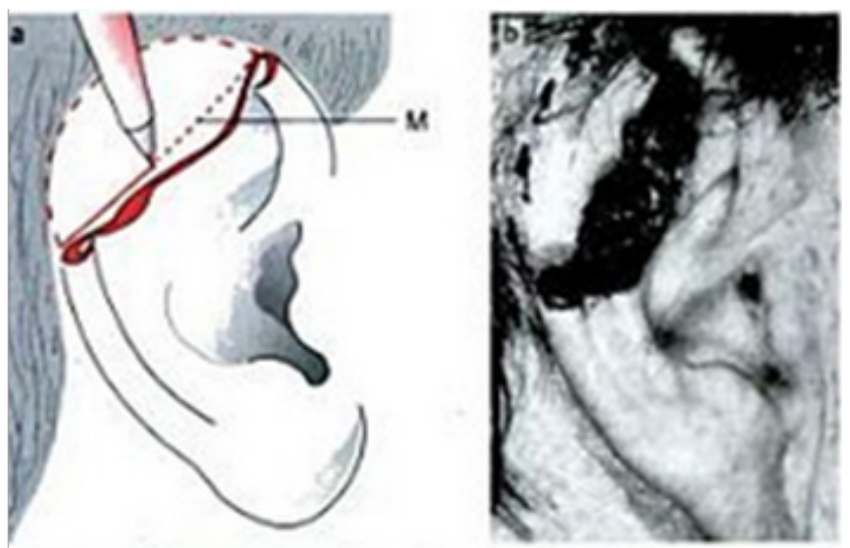

Figure 7 Marking and inscision. ${ }^{7}$
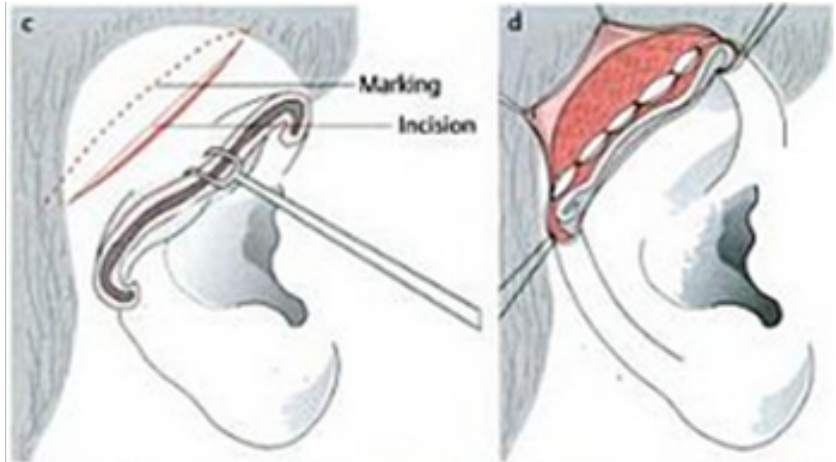

Figure 8 Developed subcutaneous pocket. ${ }^{7}$
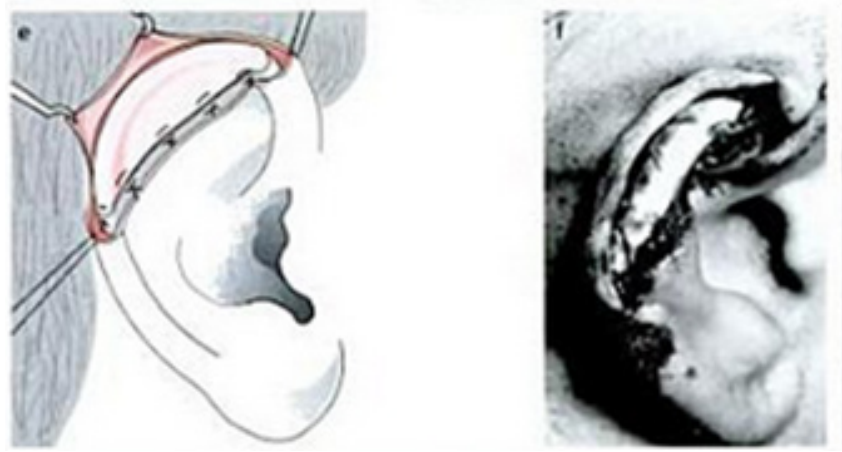

Figure 9 Attachment of the framework. ${ }^{7}$
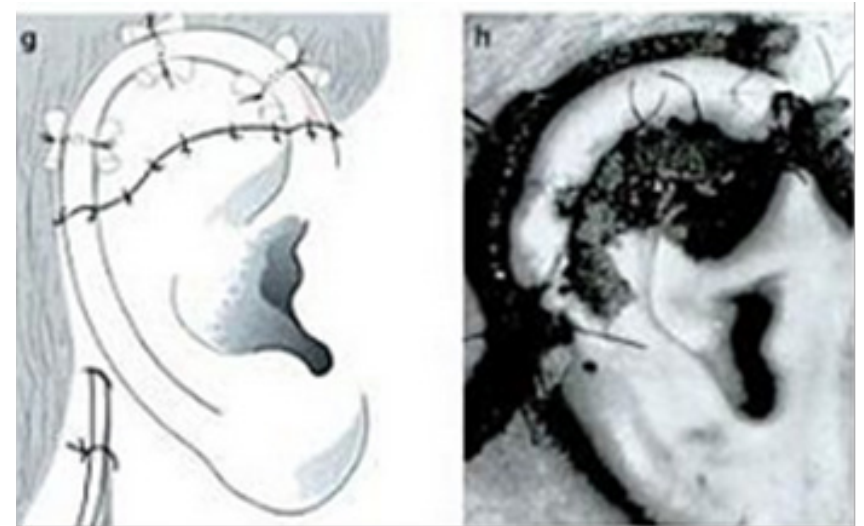

Figure 10 Closure of the skin wound.

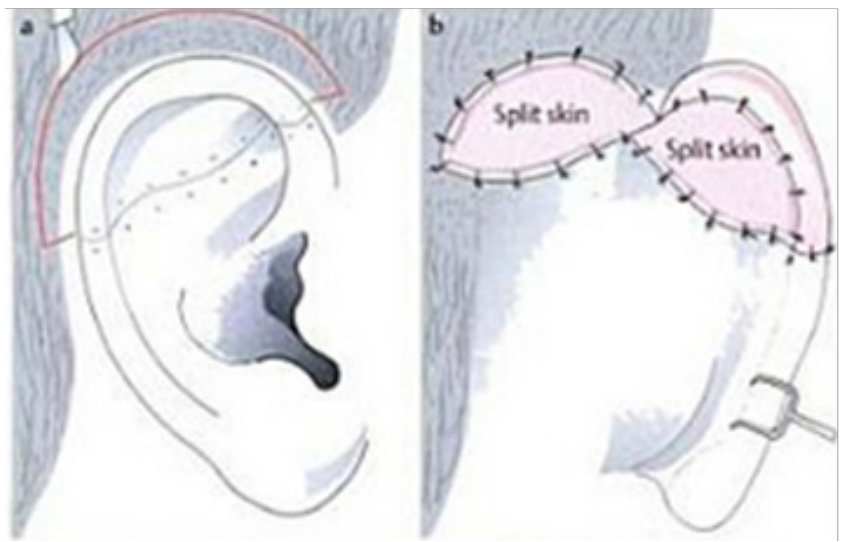

Figure II Second stage of the reconstruction of the upper auricle with Nagata Technique. 


\section{Conclusion}

Upper third auricular surgery for cauliflower ear is not only present for the appearance issue but also beneficial for eradicating bacterial pathogens. The goal of this treatment is to restore the aesthetic subunit of the auricle. On the upper third defect, the surgery techniques depend on the size and location. The greater the defect, the more components are needed to repair the auricle. Further research and literature are needed on the best techniques for every cauliflower ear cases and minimizing side effects.

\section{Acknowledgments}

We wish to acknowledge Faculty of Medicine University Indonesia and Dr. Cipto Mangunkusumo Hospital, as the place this case surgery was carried out and the paper was written. Futhermore, we would like to express our deepest gratitude to Trimartani Koento and Dini $\mathrm{W}$. Widodo for their knowledge and guidance that have given our inspiration in writing this paper.

\section{Conflicts of interest}

The author declares that there is no conflict of interest to disclose.

\section{Funding}

None.

\section{References}

1. Manninen I, Blomgren K, Elokiuru R, et al. Cauliflower ear among Finnish high-level male wrestlers and judokas is prevalent and symptomatic deformity. Scandinavian Journal of Medicine \& Science in Sports. 2019;29(12):1952-1956.

2. Noormohammadpour P, Rostami M, Nourian R, et al. Association between hearing loss and cauliflower ear in wrestlers, a Case Control Study Employing Hearing Tests. Asian Journal of Sports Medicine. 2015;6(2).
3. Yahalom S. Perichondritis: a complication of piercing auricular cartilage. Postgraduate Medical Journal. 2003;79(927):29-29.

4. Hanif J. Lesson of the week: "High" ear piercing and the rising incidence of perichondritis of the pinna. BMJ. 2001;322(7291):906-907.

5. Yotsuyanagi T, Yamashita K, Urushidate S, et al. Surgical correction of cauliflower ear. British Journal of Plastic Surgery. 2002;55(5):380-386.

6. Siegert R, Magritz R. Reconstruction of the auricle. GMS Current Topics in Otorhinolaryngology - Head and Neck Surgery. 2007;6.

7. Weerda H. Surgery of the auricle. Germany: Thieme; 2007.

8. Paper ID. Fascial plastic reconstructive $2^{\text {nd }}$ ed. New York: Thieme; 2002.

9. Byron JB, Johnson JT, Newlands SD. Head and neck surgery. $4^{\text {th }}$ ed. Lippincot william \& wilkins: 2006; p. 1167-81.

10. Davidi E, Paz A, Duchman D, et al. Perichondritis of the auricle: analysis of 114 cases. IMAJ. 2011;13(1):21-24.

11. Prasad HK, Shreedharan S, Prasad HS. Meyyuappaan MH, Harsha $\mathrm{KS}$. Perichondritis of the auricle and its management. $J$ Laryngol Otol. 2007;121(6):530-534.

12. Schonauer E, La Rusca I, Pereira JA, Molea G. Redefinition of the helical rim in cauliflower-ear surgery. British J of Plast Surg. 2002;55(1):66-86.

13. El-Bestar MF. Surgical Treatment of Cauliflower Ear Egypt. J Plast Reconstr Surg. 2004;28(1):23-27.

14. Fujiwara M, Suzuki A, Nagata T, et al. Cauliflower ear dissection. J Plast Reconstruct Aes Surg. 2011;64:279-82.

15. Kobus K, Szczyt M, Latkowski I, et al. Reconstruction of the auricle. British J Plast Surg. 2002;55:645-551.

16. Armin BB, Ruder RO, Azizadeh. Partial Auricular Reconstruction. Semin plast surg. 2011;25(4):249-56. 